Research Article

\title{
Development of Attenuated Total Reflectance Mid-Infrared (ATR-MIR) and Near-Infrared (NIR) Spectroscopy for the Determination of Resistant Starch Content in Wheat Grains
}

\author{
Rong Wang, ${ }^{1}$ Xia Wei, ${ }^{1,2}$ Hongpan Wang, ${ }^{1}$ Linshu Zhao, ${ }^{3}$ Cengli Zeng, ${ }^{4}$ Bingrui Wang, \\ Wenying Zhang, ${ }^{1}$ Luxiang Liu $\left(\mathbb{B},{ }^{3}\right.$ and Yanhao $\mathrm{Xu}\left(\mathbb{1}^{2}\right.$ \\ ${ }^{1}$ Hubei Key Laboratory of Waterlogging Disaster and Agriculture Use of Wetland and Hubei Collaborative \\ Innovation Centre for Grain Industry and Engineering Research Center of Ecology and Agriculture Use of Wetland, \\ Ministry of Education, Yangtze University, Jingzhou, Hubei 434025, China \\ ${ }^{2}$ Hubei Key Laboratory of Food Crop Germplasm and Genetic Improvement, Food Crops Institute, \\ Hubei Academy of Agricultural Sciences, Wuhan 430064, China \\ ${ }^{3}$ Institute of Crop Sciences, Chinese Academy of Agricultural Sciences, Beijing 100081, China \\ ${ }^{4}$ Hubei Engineering Research Center for Protection and Utilization of Special Biological Resources in the Hanjiang River Basin, \\ Jianghan University, Wuhan 430056, China \\ ${ }^{5}$ College of Plant Science \& Technology, Huazhong Agricultural University, Wuhan 430064, China
}

Correspondence should be addressed to Luxiang Liu; liuluxiang@caas.cn and Yanhao Xu; xyh09@yangtzeu.edu.cn

Received 7 February 2021; Revised 5 May 2021; Accepted 24 June 2021; Published 15 July 2021

Academic Editor: Alessandro Buccolieri

Copyright (C) 2021 Rong Wang et al. This is an open access article distributed under the Creative Commons Attribution License, which permits unrestricted use, distribution, and reproduction in any medium, provided the original work is properly cited.

\begin{abstract}
The chemical method for the determination of the resistant starch (RS) content in grains is time-consuming and labor intensive. Near-infrared (NIR) and attenuated total reflectance mid-infrared (ATR-MIR) spectroscopy are rapid and nondestructive analytical techniques for determining grain quality. This study was the first report to establish and compare these two spectroscopic techniques for determining the RS content in wheat grains. Calibration models with four preprocessing techniques based on the partial least squares (PLS) algorithm were built. In the NIR technique, the mean normalization + Savitzky-Golay smoothing (MN + SGS) preprocessing technique had a higher coefficient of determination $\left(R_{c}^{2}=0.672 ; R_{p}^{2}=0.552\right)$ and a relative lower root mean square error value $(\mathrm{RMSEC}=0.385$; RMSEP $=0.459)$. In the ATR-MIR technique, the baseline preprocessing method exhibited a better performance regarding to the values of coefficient of determination $\left(R_{c}^{2}=0.927 ; R_{p}^{2}=0.828\right)$ and mean square error value $(\mathrm{RMSEC}=0.153$; RMSEP $=0.284)$. The validation of the developed best NIR and ATR-MIR calibration models showed that the ATR-MIR best calibration model has a better RS prediction ability than the NIR best calibration model. Two high grain RS content wheat mutants were screened out by the ATR-MIR best calibration model from the wheat mutant library. There was no significant difference between the predicted values and chemical measured values in the two high RS content mutants. It proved that the ATR-MIR model can be a perfect substitute in RS measuring. All the results indicated that the ATR-MIR spectroscopy with improved screening efficiency can be used as a fast, rapid, and nondestructive method in high grain RS content wheat breeding.
\end{abstract}

\section{Introduction}

Resistant starch (RS) is the starch that cannot be converted into glucose when passing through the healthy small intestine [1]. Owing to its indigestion, RS can increase satiety and reduce calorie intake, which could reduce postprandial blood glucose levels [2], regulate the intestinal metabolism [3], reduce colon cancer risk [4], control bodyweight [5], and absorb minerals [6]. Due to its benefits to human health, the RS studies have attracted considerable attentions and 
promoted to be one of the important discoveries about the relationship between carbohydrates and human health $[1,2,7]$. It is also becoming a hot topic for function food breeding [7-9].

Starch in grains is the major source of carbohydrates in the human diet. The improvement of the RS content in grains is an important goal for breeding. A few high RS content grain varieties, such as RS111 [10], the hulless barley variety Himalaya 292 [11], and durum wheat [12], have been released to the public. While, they still cannot satisfy the growing demand. Induced mutagenesis and selection of natural mutations are still the major approaches for the breeding of high RS varieties [7, 13-17]. Currently, enzyme hydrolysis and the chromogenic method are the commonly used methods for RS measurement. These methods are destructive, time-consuming, costly, and cumbersome, thus delaying the process of research in RS [18]. A simple, fast, and nondestructive method for screening mutants with millions of mutants becomes vital for breeding.

Infrared spectroscopy, including near-infrared (NIR, 950-1650 $\mathrm{nm}$ ) and attenuated total reflectance mid-infrared (ATR-MIR, 525-4000 $\mathrm{cm}^{-1}$ ) spectroscopy, has been widely used as a simple, fast, and reliable substitute to conventional methods in discrimination of chemical composition [19-21]. MIR spectra can identify the fundamental vibrational absorption of functional groups in the mid-infrared region $\left(525-4000 \mathrm{~cm}^{-1}\right)$, while the NIR spectra in the range of $950 \mathrm{~nm}$ and $1650 \mathrm{~nm}$ identify overtone information and combinations of these vibrations [19-21]. The NIR technique was adopted as an official method for the prediction of crude proteins in wheat grains by the American Association of Cereal Chemists (AACC) [22]. A model based on NIR spectroscopy has been expanded to the investigation of the amylose content [23], lipid content [24], water content [25], and deoxynivalenol content in durum wheat [26] and for monitoring the wheat gluten enzyme [27]. Meanwhile, the MIR spectroscopy models have been used to analyze sugars in barley $[19,28]$, ash and moisture content in soybean [24], and proteins and lipids in different wheat varieties [29], as well as to perform nitrate determination in paddy soil [30].

Wheat (Triticum aestivum L.) feeds about $40 \%$ of the world's population [31]. Wheat grains with the high RS content could provide additional health benefits $[8,9,31]$. While, there is no spectroscopy model for determining the content of RS in wheat grains yet. Furthermore, mutation breeding is the major approach for the breeding of high RS varieties [13-17]. However, there is still no simple, fast, and nondestructive method for screening high RS mutants in breeding.

The aims of this study are to develop a simple, fast, nondestructive method for the determination of the RS content in wheat grains by using NIR and ATR-MIR spectroscopy and to apply the developed spectral methods to screen the high grain RS content wheat mutants.

\section{Materials and Methods}

2.1. Samples. Based on the rule of the range of the RS content in the calibration set should cover those in the validation set, a total of sixty-four $(n=64)$ wheat accessions were randomly divided into a calibration set (fifty-one wheat samples, Table 1) and a validation set (thirteen wheat samples, Table 2) in a ratio of $4: 1$. The calibration set and the validation set were used to develop and validate the best calibration model for the prediction of the RS content in wheat grains by NIR and ATR-MIR approaches.

The $M_{5}$ generation wheat mutant library, including 1010 mutant lines, was used to screen the high RS content mutant lines with the best calibration model from the above results. The mutants originated from wheat accession YUW-1-207 and were irradiated by a $50 \mathrm{~Gy}{ }^{7} \mathrm{Li}$ ion beam.

All wheat materials were grown at Yangtze University field stations in 2017-2018. The field trial experiments were arranged randomized with three replications for each accession. Each replicate was designed $1.2 \mathrm{~m}$ long and $0.85 \mathrm{~m}$ wide. The seeding density was kept 30 per row. Fertilization, pest, and disease control were performed on a regular basis. The analysis was only based on the plants in the middle row.

2.2. Chemical Measured RS Content. Whole grain flour was prepared from each sample by grinding in a pulveriser (Perten Laboratory Mill 3100), which was fitted with a $0.8 \mathrm{~mm}$ screen. The RS content (the amount of RS as a percentage of whole grain) was measured for $100 \mathrm{mg}$ whole grain flour using a resistant starch assay kit (K-RSTAR, Megazyme Co., Wicklow, Ireland) following the manufacturer's instructions. The sample was treated with $10 \mathrm{mg} / \mathrm{mL}$ pancreatic a-amylase and $3 \mathrm{U} / \mathrm{mL}$ amyloglucosidase (AMG) enzymes for hydrolysis and solubilization of nonresistant starch. After the enzymatic reaction was terminated by adding a $50 \%$ ethanol solution, resistant starch was recovered as a pellet by centrifugation (approx. $4000 \mathrm{r} / \mathrm{min}$, $10 \mathrm{~min}$ ). Resistant starch in the pellet was dissolved in $2 \mathrm{M}$ $\mathrm{KOH}$ before the reacted solution was repeatedly washed and decanted. Then, starch in the solution was quantitatively hydrolyzed to glucose with AMG. D-glucose was measured with glucose oxidase/peroxidase (GOPOD) reagent at $510 \mathrm{~nm}$ wavelength against the reagent blank [29]. All samples were measured with three replicates. The standard RS sample from the reagent kit was used as a control in each round of reactions.

2.3. NIR and ATR-MIR Spectroscopy. NIR spectra were collected in the range between $950 \mathrm{~nm}$ and $1650 \mathrm{~nm}$ using a DA7200 spectrometer (Perten Instruments Inc., Sweden). In the NIR method, approximately $4 \mathrm{~g}$ of wheat grains per sample was scanned in triplicate in a small ring cup. Each spectrum represented the average of 32 scans and was recorded as $\log (1 / \mathrm{R})$ at $2 \mathrm{~nm}$ increments.

MIR spectra were scanned based on a Nicolet iS5 Fourier transform infrared spectrometer (ThermoFisher Scientific, USA) with the iD7 attenuated total reflectance (ATR) accessory. The ATR-MIR spectra of each sample were obtained by taking the means of 16 scans at a resolution of $4 \mathrm{~cm}^{-1}$, in the range between 525 and $4000 \mathrm{~cm}^{-1}$, with a background of 16 scans. The air was recorded as a reference background 
TABLE 1: Wheat samples in the calibration set and the grain RS content measured by the chemical method.

\begin{tabular}{|c|c|}
\hline Wheat samples & Grain RS content (\%) \\
\hline Shannong7859 & $0.220 \pm 0.013$ \\
\hline Karagan & $0.242 \pm 0.011$ \\
\hline Baiqimai & $0.288 \pm 0.013$ \\
\hline Fan6 & $0.308 \pm 0.018$ \\
\hline Mace & $0.373 \pm 0.020$ \\
\hline Fretes-3 & $0.395 \pm 0.021$ \\
\hline Mazhamai & $0.417 \pm 0.025$ \\
\hline Dabaimai & $0.474 \pm 0.022$ \\
\hline Ganmai8hao & $0.491 \pm 0.025$ \\
\hline Huaimai16 & $0.504 \pm 0.032$ \\
\hline Zhongyou16 & $0.536 \pm 0.031$ \\
\hline Youzimai & $0.541 \pm 0.032$ \\
\hline Lumailhao & $0.549 \pm 0.033$ \\
\hline Honglidangnianlao & $0.562 \pm 0.034$ \\
\hline Sumai3hao & $0.597 \pm 0.041$ \\
\hline Xinmai19 & $0.606 \pm 0.043$ \\
\hline Jinmai2148 & $0.642 \pm 0.042$ \\
\hline Mingxian169 & $0.648 \pm 0.045$ \\
\hline Yanzhan 4110 & $0.718 \pm 0.056$ \\
\hline Jinan2hao & $0.721 \pm 0.053$ \\
\hline Jinan17hao & $0.741 \pm 0.052$ \\
\hline Jinan 13 & $0.750 \pm 0.054$ \\
\hline Geerhongmai & $1.677 \pm 0.084$ \\
\hline Zijiehong & $2.177 \pm 0.103$ \\
\hline Shuilizhan & $2.238 \pm 0.152$ \\
\hline Muzongzhuoga & $2.399 \pm 0.112$ \\
\hline Shite14 & $0.758 \pm 0.052$ \\
\hline Kord CL Plus & $0.758 \pm 0.055$ \\
\hline Xuzhou21 & $0.761 \pm 0.061$ \\
\hline Sankecun & $0.780 \pm 0.063$ \\
\hline Zheng6fu & $0.793 \pm 0.062$ \\
\hline Diyouzao & $0.847 \pm 0.065$ \\
\hline N553 & $0.892 \pm 0.061$ \\
\hline Kopara 73 & $0.910 \pm 0.062$ \\
\hline Honghuamai & $0.919 \pm 0.076$ \\
\hline Yunmai34 & $0.938 \pm 0.075$ \\
\hline Miannong4hao & $1.008 \pm 0.075$ \\
\hline Meiqianwu & $1.046 \pm 0.073$ \\
\hline Jinmai8hao (Jinzhong849) & $1.082 \pm 0.076$ \\
\hline Ningmai9hao & $1.091 \pm 0.071$ \\
\hline Wenmai6hao (Yumai49) & $1.119 \pm 0.078$ \\
\hline Jiahongmai & $1.139 \pm 0.071$ \\
\hline AUS 19399 & $1.200 \pm 0.072$ \\
\hline Liuyuehong & $1.232 \pm 0.083$ \\
\hline Jiangmai & $1.379 \pm 0.083$ \\
\hline Huzhuhong & $1.487 \pm 0.083$ \\
\hline Zang2726 & $1.533 \pm 0.086$ \\
\hline Honghuazao & $1.615 \pm 0.083$ \\
\hline Heshangmai & $2.548 \pm 0.107$ \\
\hline Wujiangzhuo & $2.601 \pm 0.155$ \\
\hline Baihuamai & $3.348 \pm 0.167$ \\
\hline
\end{tabular}

spectrum. The ATR crystal was carefully cleaned with ethanol after each sample measurement.

2.4. Data Analysis. The NIR and ATR-MIR spectra were uploaded to Unscrambler 9.7 software (CAMO Corporation, USA) for chemometric analysis. The models for the calibration between the measured values and the infrared spectra were established using partial least squares (PLS) regression with full cross-validation. The quality of the models was assessed by the determination coefficient of calibration $\left(R_{c}^{2}\right)$, the determination coefficient of prediction $\left(R_{p}^{2}\right)$, the root mean square error of calibration (RMSEC), and the root mean square error of prediction (RMSEP) [32]. Moreover, the residual predictive deviation (RPD), a statistical parameter defined as the ratio of the standard deviation (SD) to the RMSEP [30, 32], was used to assess the predictive ability of the calibration models.

The preprocessing methods were used to eliminate the interferences of background signal, random noise, and light scattering from the spectra, which can be divided into the scatter correction group and spectral derivatization group $[33,34]$. Four preprocessing methods including Gaussian filter smoothing (GFS), multiplicative scatter correction (MSC), baseline mean normalization (MN), and Savitzky-Golay smoothing (SGS) were used to transform the NIR and ATR-MIR spectra before calibration to eliminate interference noise such as baseline drift, tilt and reverse, and light scattering $[35,36]$. The MN, baseline, and MSC preprocessing methods belonged to the scatter correction group, and the SGS and GFS belong to the spectral derivatization group. The developed model, with the highest value of determination coefficient, the lowest value of root mean square error (RMSE), and the highest value of RPD, was chosen as the best calibration model.

\section{Results}

3.1. Chemical Measured Wheat Grain RS Content. The RS content in whole wheat grain flour samples was measured by the AOAC method in the calibration set ranged from $0.220 \%$ to $3.348 \%$ with the mean content $1.011 \%$ (Table 1 ); while the RS content in the validation set ranged from $0.267 \%$ to $2.842 \%$ with the mean content $1.285 \%$ (Table 2). The standard deviation (SD) in the calibration set and validation set is 0.679 and 0.697 , respectively. The coefficient of variation $(\mathrm{CV})$ in the calibration set and validation set is $67.086 \%$ and $53.846 \%$, respectively. A wide distribution of the wheat grain RS content was observed in the calibration set and validation set.

\subsection{Development of NIR and ART-MIR Prediction Models.} The ATR-MIR spectrum $\left(525-4000 \mathrm{~cm}^{-1}\right)$ (Figure 1(a)) region has strong absorption peaks and belongs to the fundamental molecular vibration modes. The peaks between 3600 and $3000 \mathrm{~cm}^{-1}$ were assigned to hydrogen bonded water $(\mathrm{O}-\mathrm{H}$ stretching vibration). The weak band detected at $1652 \mathrm{~cm}^{-1}$ was responsible for $\mathrm{C}=\mathrm{O}$ vibration of the decarboxylated groups. The region between 1200 and $950 \mathrm{~cm}^{-1}$ was attributed to the $\mathrm{O}-\mathrm{C}$ stretch vibrations of the glucose ring $[37,38] .1146 \mathrm{~cm}^{-1}$ and $1047 \mathrm{~cm}^{-1}$ are the stretching vibrations linked to the primary and secondary alcohol hydroxyl groups in glucose, and $1002 \mathrm{~cm}^{-1}$ is the $\mathrm{C}-\mathrm{O}$ stretching vibration of the pyranose ring. C-O-H, C-C$\mathrm{H}$, and $\mathrm{O}-\mathrm{C}-\mathrm{H}$ bending of the anomeric configuration of carbohydrates occurred between 750 and $950 \mathrm{~cm}^{-1}[39,40]$. 
TABLE 2: Wheat samples in the validation set and grain RS content determined by both the chemical method and the best ATR-MIR and NIR calibration models.

\begin{tabular}{|c|c|c|c|c|c|}
\hline \multirow{2}{*}{ Wheat samples } & \multicolumn{3}{|c|}{ Grain RS content (\%) } & \multicolumn{2}{|c|}{ Relative error $(\mathrm{RE})^{*}$} \\
\hline & Chemical measured & ATR-MIR predicted & NIR predicted & ATR-MIR RE & NIR RE \\
\hline Xiaokouhong & $0.267 \pm 0.011$ & $0.332 \pm 0.041$ & $0.591 \pm 0.085$ & 24.345 & 121.348 \\
\hline Yu30691-1-3 & $0.615 \pm 0.032$ & $0.423 \pm 0.032$ & $0.834 \pm 0.088$ & 31.212 & 35.610 \\
\hline Hongpidongmai & $0.784 \pm 0.041$ & $0.864 \pm 0.065$ & $1.019 \pm 0.091$ & 10.204 & 29.974 \\
\hline Tanori & $0.887 \pm 0.063$ & $0.861 \pm 0.073$ & $0.661 \pm 0.093$ & 2.931 & 25.479 \\
\hline Hongpixiaomai & $0.991 \pm 0.068$ & $1.160 \pm 0.082$ & $0.684 \pm 0.082$ & 17.053 & 30.979 \\
\hline PI94365 & $1.028 \pm 0.065$ & $0.998 \pm 0.081$ & $0.814 \pm 0.091$ & 2.918 & 20.817 \\
\hline Huadong6hao & $1.172 \pm 0.072$ & $0.892 \pm 0.093$ & $0.937 \pm 0.105$ & 23.891 & 20.051 \\
\hline Tumangmai & $1.230 \pm 0.083$ & $1.093 \pm 0.101$ & $1.506 \pm 0.132$ & 11.138 & 22.439 \\
\hline Mahon Demias & $1.365 \pm 0.085$ & $1.216 \pm 0.097$ & $1.131 \pm 0.139$ & 10.916 & 17.143 \\
\hline Chixiaomai & $1.508 \pm 0.095$ & $1.637 \pm 0.105$ & $1.054 \pm 0.108$ & 8.554 & 30.106 \\
\hline Baitiaoyu & $1.725 \pm 0.105$ & $1.487 \pm 0.127$ & $1.398 \pm 0.153$ & 13.797 & 18.957 \\
\hline Mangxiaomai & $2.288 \pm 0.112$ & $1.798 \pm 0.153$ & $1.587 \pm 0.186$ & 21.416 & 30.638 \\
\hline Lanhuamai & $2.842 \pm 0.117$ & $2.200 \pm 0.194$ & $1.851 \pm 0.225$ & 22.590 & 34.870 \\
\hline
\end{tabular}

${ }^{*} \mathrm{RE}$, the ratio between the measured value minus the predicted value divided by the measured value.

For the NIR spectra (950-1650 nm) (Figure 1(b)), two peaks were observed; the weak intensity was found round $1200 \mathrm{~nm}$, and the intense peak was found around $1500 \mathrm{~nm}$. The absorption around $1215 \mathrm{~nm}$ and $1483 \mathrm{~nm}$ was, respectively, reported to be related to the stretching of C-H and N-H [41].

The collected ATR-MIR and NIR spectra from the calibration set were used to develop calibration models through PLS regression with full cross-validation. For the NIR spectra, the model with MN + SGS preprocessing had the highest $R_{c}^{2}$ and RPD, which were 0.672 and 1.464 , respectively. At the same time, RMSE in this model reached as low as 0.459 (Table 3). The MN + SGS preprocessing was chosen as the best calibration model for the NIR spectroscopy. For the ATR-MIR spectra, the baseline preprocessing method was chosen as the best calibration model with $R_{c}^{2}$, RPD, and RMSE 0.937, 2.391, and 0.284, respectively (Table 3 ).

Meanwhile, a much better correlation of the chemical determined values and the predicted values was observed in the ATR-MIR spectroscopy model (Figure 2(a)) than in the NIR spectroscopy model (Figure 2(b)). The correlation between predicted values and real values in the ATR-MIR model was 0.937 , while the value in the NIR model was 0.672 . The results showed the ATR-MIR spectroscopy model may have a better performance in the prediction of the RS content in wheat grains than the NIR spectroscopy model.

\subsection{Validation of NIR and ATR-MIR Best Calibration Models.}

To verify the accuracy and repeatability of models with the best preprocessing techniques in two spectroscopies, validation was performed in the validation set. The experimentally determined RS values, the predicted RS values, and the calculated relative error (measurement/prediction value) are given in Table 2. For the NIR calibration model with the MN + SGS preprocessing, the relative error ranged from $17.143 \%$ to $121.348 \%$, with a mean relative error of $34.028 \%$. For the ATR-MIR calibration model with the baseline preprocessing method, the relative error ranged from $2.931 \%$ to $31.220 \%$, with a mean relative error of $15.832 \%$. A linear regression analysis was performed between the measured value and the value predicted by the ATR-MIR model $\left(R^{2}=0.919\right) \quad$ (Figure 3(a)) and the NIR model $\left(R^{2}=0.773\right)$ (Figure $\left.3(\mathrm{~b})\right)$. The results confirmed that the ATR-MIR spectroscopy provided a better performance than NIR spectroscopy for the prediction of the RS content in wheat grains.

\subsection{Screen of High RS Wheat Mutants by the ATR-MIR Model.} To check the application of the developed ATR-MIT model, the best ATR-MIR calibration model with baseline preprocessing was promoted to predict the grain RS content of 1010 wheat mutants (Figure 4). The predicted RS content of 1010 wheat mutants ranged from $0.101 \pm 0.018 \%$ to $2.553 \pm 0.311 \%$. Two lines with YUW-RSH1 $(2.553 \pm 0.311 \%)$ and YUW-RSH2 $(2.116 \pm 0.230 \%)$ highest RS content were identified (Table 4). At the same time, the RS content was also validated by the chemical method. The chemical determined RS content in YUW-RSH1 and YUWRSH2 was $2.572 \pm 0.090 \%$ and $2.126 \pm 0.071 \%$, respectively (Table 4). There was no significant difference between the predicted values and chemical determined values. The results showed that ATR-MIR spectroscopy can be an effective way to screen and identify high grain RS content materials for wheat breeding.

\section{Discussion}

4.1. ATR-MIR Spectroscopy Had a Better Performance for the Prediction of the RS Content in Wheat Grains than NIR Spectroscopy. The MIR and NIR spectroscopies have become the fastest growing and most compelling modern quantitative analysis technologies for assessment of the quality of both macronutrients and minor compounds in agricultural products and food [21, 27, 42, 43]. In agriculture, protein, tannins, lipids, phytic acid, and most of the amino acids were the commonly detected components in crops [23, 27-29,34]. In this study, a total of 64 wheat samples were used to develop the ATR-MIR and NIR 


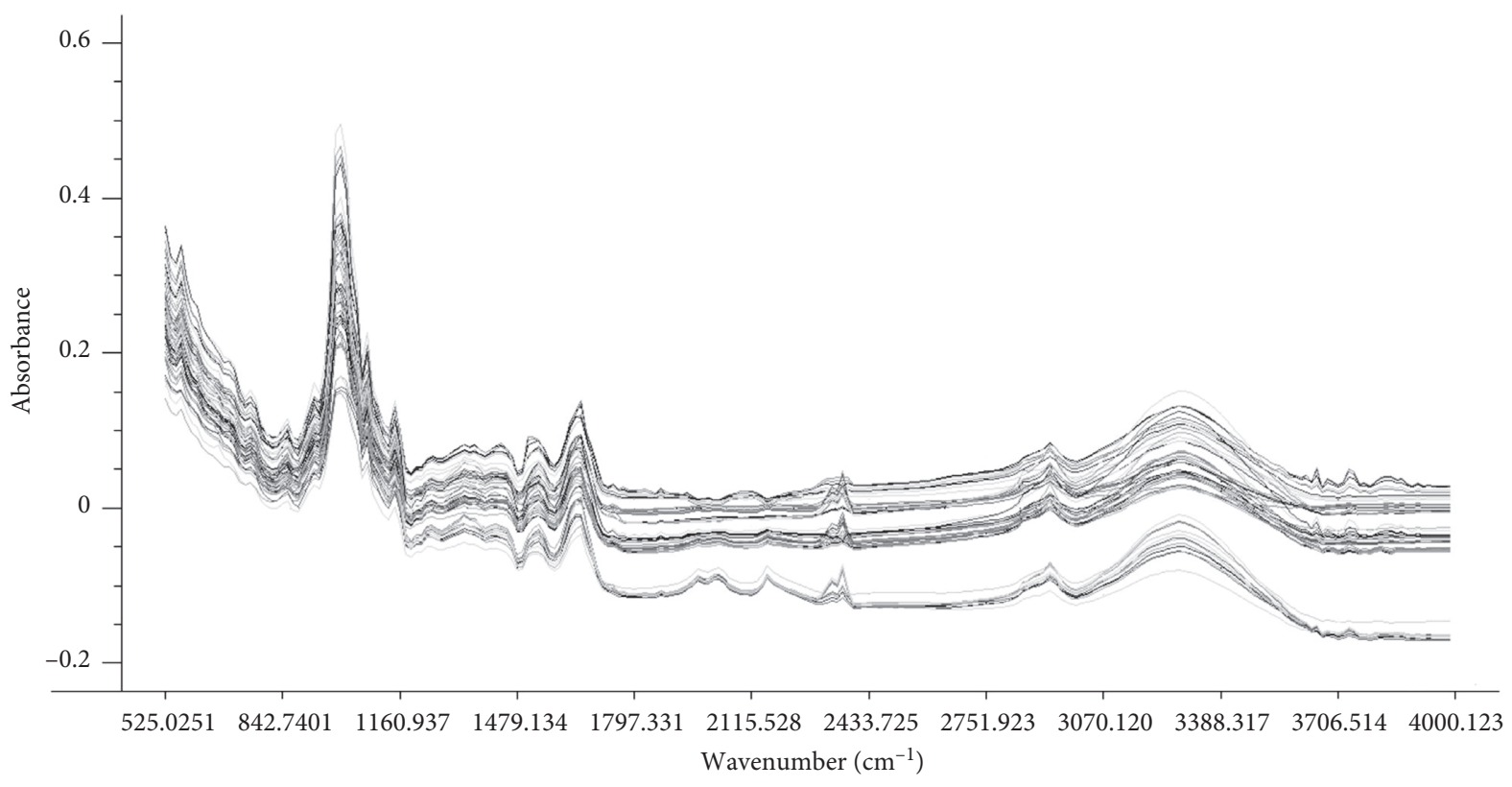

(a)

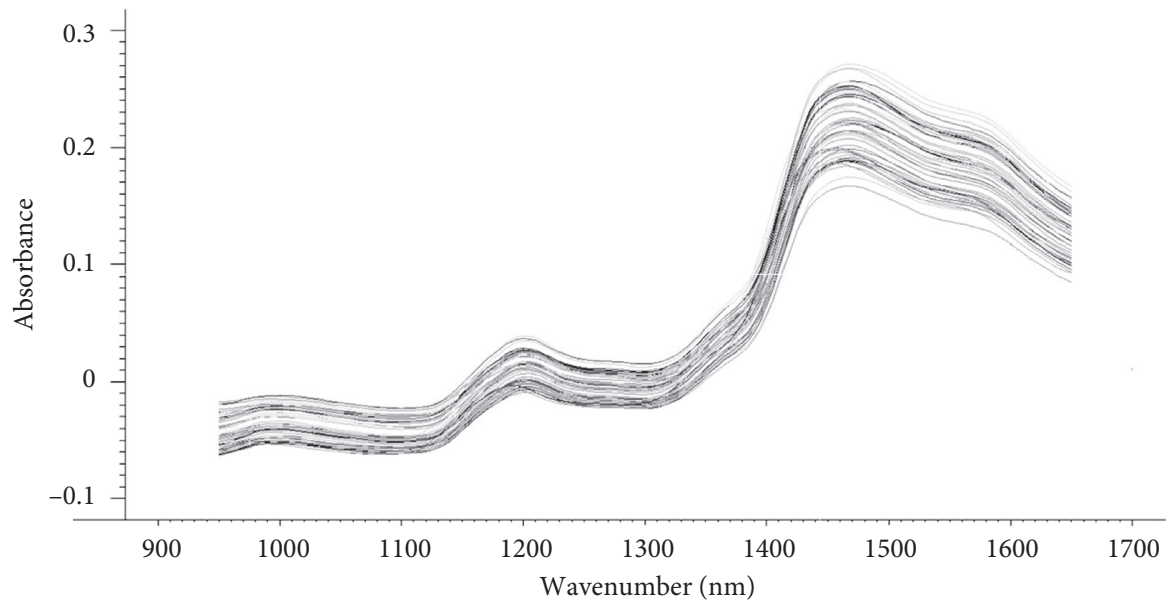

(b)

FIGURE 1: ATR-MIR and NIR spectra of wheat grain samples obtained in this study. (a) ATR-MIR spectra obtained in the range between 525 and $4000 \mathrm{~cm}^{-1}$ without pretreatment. (b) NIR spectra obtained in the range between 950 and $1650 \mathrm{~nm}$ without pretreatment.

TABLE 3: Develop and screening for the best calibration model for resistant starch in wheat grain samples using ATR-MIR and NIR spectra.

\begin{tabular}{|c|c|c|c|c|c|c|}
\hline \multirow{2}{*}{ Spectroscopy } & \multirow{2}{*}{ Preprocessing methods } & \multicolumn{2}{|c|}{ Calibration } & \multicolumn{3}{|c|}{ Internal cross-validation } \\
\hline & & $R_{c}^{2}$ & RMSEC & $R_{p}^{2}$ & RMSEP & $\mathrm{RPD}$ \\
\hline \multirow{4}{*}{ NIR } & Original & 0.641 & 0.403 & 0.482 & 0.493 & 1.363 \\
\hline & MN & 0.673 & 0.384 & 0.526 & 0.472 & 1.424 \\
\hline & $\mathrm{MN}+\mathrm{MSC}$ & 0.671 & 0.385 & 0.523 & 0.474 & 1.418 \\
\hline & $\mathrm{MN}+\mathrm{SGS}$ & 0.672 & 0.385 & 0.552 & 0.459 & 1.464 \\
\hline \multirow{4}{*}{ ATR-MIR } & Original & 0.922 & 0.188 & 0.804 & 0.303 & 2.218 \\
\hline & Baseline & 0.937 & 0.169 & 0.828 & 0.284 & 2.366 \\
\hline & Baseline + GFS & 0.935 & 0.171 & 0.826 & 0.286 & 2.35 \\
\hline & Baseline + SGS & 0.935 & 0.171 & 0.825 & 0.287 & 2.341 \\
\hline
\end{tabular}

$R_{c}^{2}$, determination coefficient of calibration; $R_{p}^{2}$, determination coefficient of prediction; RMSEC, root mean square error of calibration; RMSEP, root mean square error of prediction; RPD, residual predictive deviation; MN, mean normalization; MSC, multiplicative scatter correction; SGS, Savitzky-Golay smoothing; GFS, Gaussian filter smoothing. 


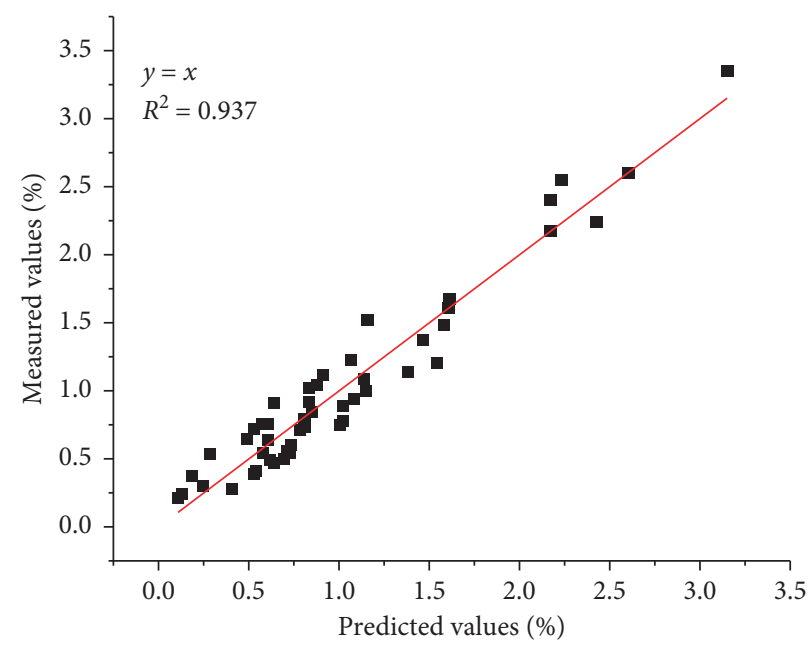

(a)

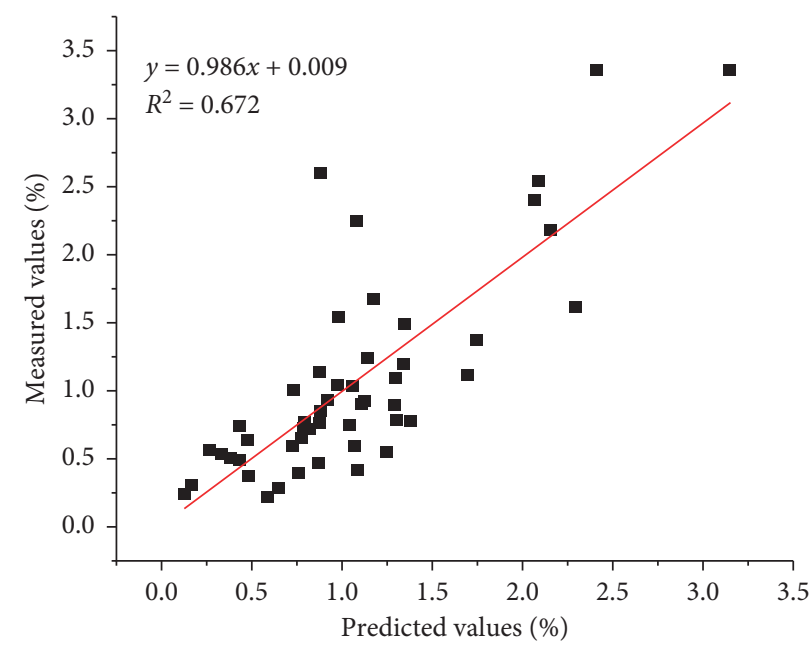

(b)

FIGURE 2: Relation between the measured values and the predicted values for the grain resistance starch content by the calibration models obtained by ATR-MIR and NIR. (a) The calibration model obtained by ATR-MIR. (b) The calibration model obtained by NIR.

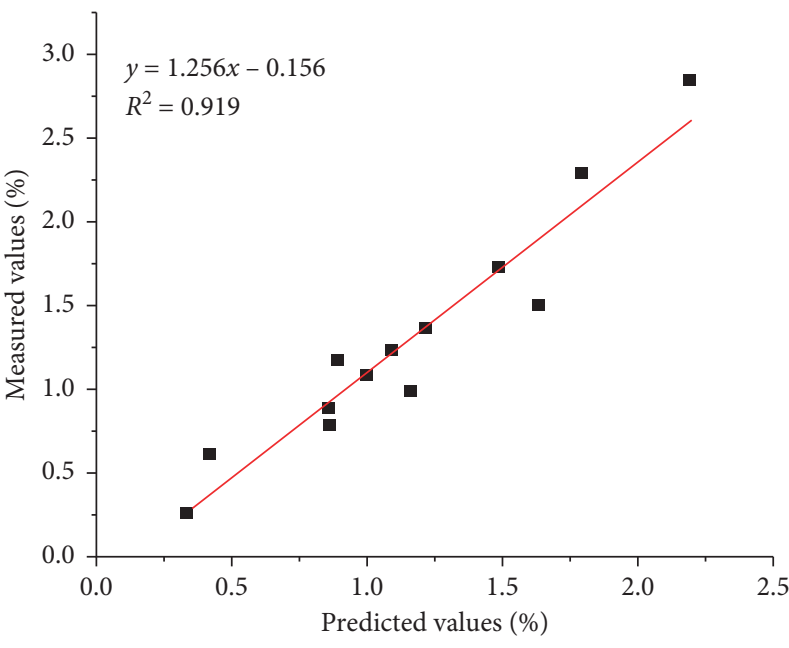

(a)

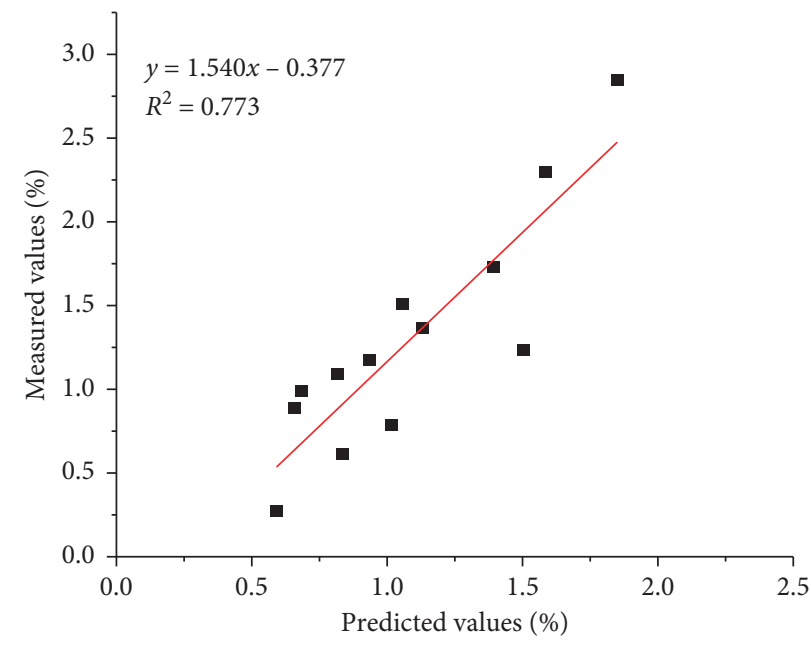

(b)

FIgURE 3: The external validation of the best ATR-MIR and NIR models. (a) The ATR-MIR model using the baseline preprocessing method. (b) The NIR model using the MN + SGS pretreatment method.

calibration models for the prediction of the RS content in wheat grains. Compared with previous studies, the samples and the distribution of the wheat grain RS content in this study have reached the requirements of infrared basic modeling [20, 24, 42].

It is commonly accepted that regression models with $R^{2}$ above 0.91 are regarded as excellent, $R^{2}=0.82-0.9$ indicated good predictive ability; $R^{2}=0.50-0.65$ reveals approximate quantitative performance [44]; and when RPD was greater than 2, the models were considered excellent, whereas values lower than 1.5 indicate not enough for applications [45]. In our results, the best NIR models showed an approximate quantitative performance $\left(R_{c}^{2}=0.672 ; R_{p}^{2}=0.552 ; \mathrm{RPD}=1.464\right)$, and the best MIR model gave a good prediction performance $\left(R_{c}^{2}=0.927\right.$; $\left.R_{p}^{2}=0.828 ; \mathrm{RPD}=2.366\right)$. Overall, the ATR-MIR displayed a better performance for the evaluation of the wheat grain RS content.

Previous reports comparing the ATR-MIR and NIR techniques for the measurement of chemical differences and quantitative analysis of substances showed that the NIR and MIR techniques have different prediction effects $[21,24,42,46]$. In soybean samples, the NIR technique was suggested for the prediction of protein and lipid determination, while the MIR technique was suggested for ash and moisture determination [24]. In rice samples, the NIR technique and the MIR technique were the best predictors of starch and protein, respectively [46]. In this study, we found that the ATR-MIR spectroscopy had a better performance for the prediction of the RS content in wheat grains than NIR spectroscopy based on the PLS regression. Nowadays, the recent developed deep learning and artificial intelligence 


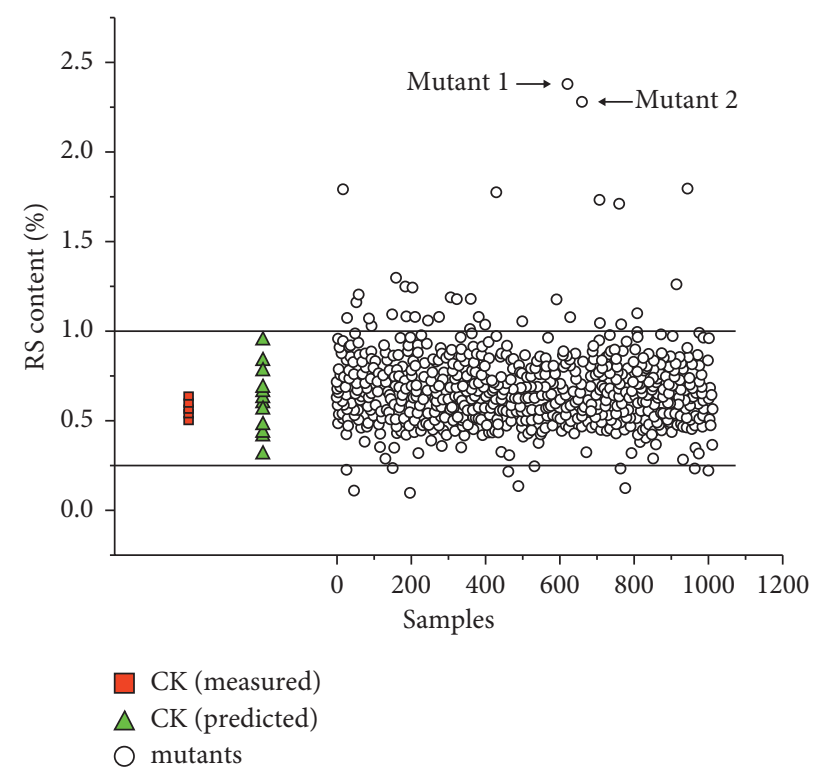

Figure 4: Application of the best ATR-MIR model to screen the high grain RS content mutant lines from wheat mutant library.

TABLE 4: Verification of the best ATR-MIR calibration model screened high grain RS wheat mutants by the chemical measured method.

\begin{tabular}{lcccc}
\hline & \multicolumn{2}{c}{ YUW-RSH1 } & \multicolumn{2}{c}{ YUW-RSH2 } \\
& ATR-MIR predicted (\%) & Chemical measured (\%) & ATR-MIR predicted (\%) & Chemical measured (\%) \\
\hline Replication 1 & 2.623 & 2.487 & 2.378 & 2.121 \\
Replication 2 & 2.245 & 2.66 & 1.945 & 2.199 \\
Replication 3 & 2.791 & 2.569 & 2.025 & 2.058 \\
Variance analysis & $2.553 \pm 0.311 \mathrm{a}$ & $2.572 \pm 0.090 \mathrm{a}$ & $2.116 \pm 0.230 \mathrm{a}$ & $2.126 \pm 0.071 \mathrm{a}$ \\
\hline
\end{tabular}

algorithm could be used to improve the stability and robustness of the spectral model, which may also become trend of the future research [47].

4.2. Rapid RS Content Assessment Method for Wheat Breeding. To date, mutation breeding is the major approach for the breeding of high RS varieties [7, 13-17]. Rapid quality assessment methods are taking an increasing important role in breeding programs; this is especially the case in mutation breeding. In this study, the developed best calibration ATR-MIR model was used to screen out 2 high resistance starch wheat mutants from the mutant library. Comparing with the chemical methods with 24 hours' time cost for each sample, the total time for the spectroscopic method just needs less than five minutes per sample, which provided the possibility to screening tens of thousands of breeding materials. The spectroscopic method could greatly improve the measurement efficiency and provide a new approach for crop resistant starch breeding and research.

\section{Conclusion}

MIR and NIR techniques were demonstrated to be useful for the prediction of the RS content in wheat grains. The ATRMIR technique provided a better predictive ability than the NIR technique. The total time required for the measurement of each sample was less than 5 minutes, compared to approximately 20 hours required for the determination of the RS content by chemical methods. In addition, we confirmed that the use of ATR-MIR spectroscopy to assist in the screening and identification of the wheat RS content was an effective approach.

\section{Data Availability}

Majority of the data used to support the findings of this study are included within the article. Other data are made available from the first author and corresponding authors upon request.

\section{Conflicts of Interest}

The authors declare that there are no conflicts of interest.

\section{Authors' Contributions}

YX and LL conceived and designed the experiments. XW, RW, and LZ performed the experiments, XW, YX, RW, HW, $\mathrm{WZ}, \mathrm{CZ}$, and BW performed data analysis, YX, XW, RW, and LL wrote the manuscript. All authors read and approved the manuscript. Rong Wang and Xia Wei contributed equally to this work. 


\section{Acknowledgments}

This work was carried out with the financial support from the National Key Research and Development Program (2016YFD0102101), Open Research Fund of Hubei Engineering Research Center for Protection and Utilization of Special Biological Resources in the Hanjiang River Basin (202008), and Open Research Fund of Engineering Research Center of Ecology and Agriculture Use of Wetland (KFT202007).

\section{References}

[1] A. Genoni, C. T. Christophersen, J. Lo et al., "Long-term Paleolithic diet is associated with lower resistant starch intake, different gut microbiota composition and increased serum TMAO concentrations," European Journal of Nutrition, vol. 59, no. 5, pp. 1845-1858, 2020.

[2] A. Kumar, U. Sahoo, B. Baisakha et al., "Resistant starch could be decisive in determining the glycemic index of rice cultivars," Journal of Cereal Science, vol. 79, pp. 348-353, 2018.

[3] S. Hald, A. G. Schioldan, M. E. Moore et al., "Effects of arabinoxylan and resistant starch on intestinal microbiota and short-chain fatty acids in subjects with metabolic syndrome: a randomised crossover study," PLoS One, vol. 11, no. 7, Article ID e $0159223,2016$.

[4] H. Yuan, X. Zhu, D. Chen, W. Wang, S. Meng, and J. Wang, "Effects of dual modified resistant indica rice starch on azoxymethane-induced incipient colon cancer in mice," Experimental and Therapeutic Medicine, vol. 13, no. 5, pp. 2036-2042, 2017.

[5] M. J. Keenan, J. Zhou, K. L. McCutcheon et al., "Effects of resistant starch, A non-digestible fermentable fiber, on reducing body fat," Obesity, vol. 14, no. 9, pp. 1523-1534, 2006.

[6] P. Raigond, R. Ezekiel, and B. Raigond, "Resistant starch in food: a review," Journal of the Science of Food and Agriculture, vol. 95, no. 10, pp. 1968-1978, 2015.

[7] K. Petropoulou, L. J. Salt, C. H. Edwards et al., "A natural mutation in Pisum sativum L. (pea) alters starch assembly and improves glucose homeostasis in humans," Nature Food, vol. 1, no. 11, pp. 693-704, 2020.

[8] D. Lafiandra, G. Riccardi, and P. R. Shewry, "Improving cereal grain carbohydrates for diet and health," Journal of Cereal Science, vol. 59, no. 3, pp. 312-326, 2014.

[9] S. O. Hansson, P. Åman, W. Becker et al., "Breeding for public health: a strategy," Trends in Food Science \& Technology, vol. 80, pp. 131-140, 2018.

[10] C. Z. Yang, X. L. Shu, L. L. Zhang et al., "Starch properties of mutant rice high in resistant starch," Journal of Agricultural and Food Chemistry, vol. 54, no. 2, pp. 523-528, 2006.

[11] D. L. Topping, M. K. Morell, R. A. King, Z. Li, A. R. Bird, and M. Noakes, "Resistant starch and health-himalaya 292, a novel barley cultivar to deliver benefits to consumers," Starch-Stärke, vol. 55, no. 12, pp. 539-545, 2003.

[12] B. Hazard, X. Zhang, M. Naemeh, and J. Dubcovsky, "Registration of durum wheat germplasm lines with combined mutations in SBEII a and SBEIIb genes conferring increased amylose and resistant starch," Journal of Plant Registrations, vol. 8, no. 3, pp. 334-338, 2014.

[13] E. Botticella, F. Sestili, A. Hernandez-Lopez, A. Phillips, and D. Lafiandra, "High resolution melting analysis for the detection of EMS induced mutations in wheat SbeIIa genes," BMC Plant Biology, vol. 11, no. 1, p. 156, 2011.
[14] B. Hazard, X. Zhang, P. Colasuonno et al., "Induced mutations in the starch branching enzyme II (SBEII) genes increase amylose and resistant starch content in durum wheat," Crop Science, vol. 52, no. 4, pp. 1754-1766, 2012.

[15] X. Shu, J. Xu, Y. Wang, S. K. Rasmussen, and D. Wu, "Effects of gamma irradiation on starch digestibility of rice with different resistant starch content," International Journal of Food Science \& Technology, vol. 48, no. 1, pp. 35-43, 2013.

[16] A. Mishra, A. Singh, M. Sharma, P. Kumar, and J. Roy, "Development of EMS-induced mutation population for amylose and resistant starch variation in bread wheat (Triticum aestivum) and identification of candidate genes responsible for amylose variation," BMC Plant Biology, vol. 16, no. 1 , p. 217, 2016.

[17] A. Schönhofen, X. Zhang, and J. Dubcovsky, "Combined mutations in five wheat STARCH branching enzyme II genes improve resistant starch but affect grain yield and breadmaking quality," Journal of Cereal Science, vol. 75, pp. 165174, 2017.

[18] A. Perera, V. Meda, and R. T. Tyler, "Resistant starch: a review of analytical protocols for determining resistant starch and of factors affecting the resistant starch content of foods," Food Research International, vol. 43, no. 8, pp. 1959-1974, 2010.

[19] D. Cozzolino, S. Roumeliotis, and J. Eglinton, "Feasibility study on the use of attenuated total reflectance MIR spectroscopy to measure the fructan content in barley," Analytical Methods, vol. 6, no. 19, pp. 7710-7715, 2014.

[20] D. Cozzolino, S. Degner, and J. Eglinton, "A novel approach to monitor the hydrolysis of barley (hordeum vulgare L) malt: a chemometrics approach," Journal of Agricultural and Food Chemistry, vol. 62, no. 48, pp. 11730-11736, 2014.

[21] B. Carbas, N. Machado, D. Oppolzer et al., "Comparison of near-infrared (NIR) and mid-infrared (MIR) spectroscopy for the determination of nutritional and antinutritional parameters in common beans," Food Chemistry, vol. 306, Article ID 125509, 2020.

[22] B. G. Osborne, T. Fearn, and P. H. Hindle, "Practical NIR spectroscopy with applications in food and beverage analysis," in Book Practical NIR Spectroscopy with Applications in Food and Beverage AnalysisLongman scientific and technical, London, UK, 1993.

[23] P. S. Sampaio, A. Soares, A. Castanho, A. S. Almeida, J. Oliveira, and C. Brites, "Optimization of rice amylose determination by NIR-spectroscopy using PLS chemometrics algorithms," Food Chemistry, vol. 242, pp. 196-204, 2018.

[24] D. S. Ferreira, O. F. Galão, J. A. L. Pallone, and R. J. Poppi, "Comparison and application of near-infrared (NIR) and mid-infrared (MIR) spectroscopy for determination of quality parameters in soybean samples," Food Control, vol. 35, no. 1, pp. 227-232, 2014.

[25] I. Torres, M.-T. Sánchez, M. Benlloch-González, and D. PérezMarín, "Irrigation decision support based on leaf relative water content determination in olive grove using near infrared spectroscopy," Biosystems Engineering, vol. 180, pp. 50-58, 2019.

[26] V. Lippolis, M. Pascale, S. Cervellieri, A. Damascelli, and A. Visconti, "Screening of deoxynivalenol contamination in durum wheat by MOS-based electronic nose and identification of the relevant pattern of volatile compounds," Food Control, vol. 37, pp. 263-271, 2014.

[27] Y. Zhang, L. Luo, J. Li et al., "In-situ and real-time monitoring of enzymatic process of wheat gluten by miniature fiber NIR spectrometer," Food Research International, vol. 99, pp. 147-154, 2017. 
[28] Y. Huang, J. Carragher, and D. Cozzolino, "Measurement of fructose, glucose, maltose and sucrose in barley malt using attenuated total reflectance mid-infrared spectroscopy," Food Analytical Methods, vol. 9, no. 4, pp. 1079-1085, 2016.

[29] K. Sujka, P. Koczoń, A. Ceglińska, M. Reder, and H. Ciemniewska-Żytkiewicz, "The application of FT-IR spectroscopy for quality control of flours obtained from polish producers," Journal of Analytical Methods in Chemistry, vol. 2017, Article ID 4315678, 9 pages, 2017.

[30] B. V. McCleary, M. McNally, P. Rossiter et al., "Measurement of resistant starch by enzymatic digestion in starch and selected plant materials: collaborative study," Journal of AOAC International, vol. 85, no. 5, pp. 1103-1111, 2002.

[31] B. Hazard, K. Trafford, A. Lovegrove, S. Griffiths, C. Uauy, and P. Shewry, "Strategies to improve wheat for human health," Nature Food, vol. 1, no. 8, pp. 475-480, 2020.

[32] Y. Shao, C. Du, J. Zhou et al., "Quantitative analysis of different nitrogen isotope labelled nitrates in paddy soil using mid-infrared attenuated total reflectance spectroscopy," $A n$ alytical Methods, vol. 9, no. 36, pp. 5388-5394, 2017.

[33] R. J. Barnes, M. S. Dhanoa, and S. J. Lister, "Standard normal variate transformation and de-trending of near-infrared diffuse reflectance spectra," Applied Spectroscopy, vol. 43, no. 5, pp. 772-777, 1989.

[34] P. Geladi, D. MacDougall, and H. Martens, "Linearization and scatter-correction for near-infrared reflectance spectra of meat," Applied Spectroscopy, vol. 39, no. 3, pp. 491-500, 1985.

[35] C. M. McGoverin, A. S. S. Clark, S. E. Holroyd, and K. C. Gordon, "Raman spectroscopic quantification of milk powder constituents," Analytica Chimica Acta, vol. 673, no. 1, pp. 26-32, 2010.

[36] M. R. Almeida, K. d. S. Oliveira, R. Stephani, and L. F. C. de Oliveira, "Fourier-transform Raman analysis of milk powder: a potential method for rapid quality screening," Journal of Raman Spectroscopy, vol. 42, no. 7, pp. 1548-1552, 2011.

[37] D. Damiran and P. Yu, "Molecular basis of structural makeup of hulless barley in relation to rumen degradation kinetics and intestinal availability in dairy cattle: a novel approach," Journal of Dairy Science, vol. 94, no. 10, pp. 5151-5159, 2011.

[38] X. Zhang and P. Yu, "Using ATR-FT/IR molecular spectroscopy to detect effects of blend DDGS inclusion level on the molecular structure spectral and metabolic characteristics of the proteins in hulless barley," Spectrochimica Acta Part A: Molecular and Biomolecular Spectroscopy, vol. 95, pp. 53-63, 2012.

[39] B. Stuart and D. J. Ando, "Modern infrared spectroscopy: analytical chemistry by open learning," in Book Modern Infrared Spectroscopy: Analytical Chemistry by Open LearningWiley Greenwich, London, UK, 1996.

[40] S. Grassi, J. M. Amigo, C. B. Lyndgaard, R. Foschino, and E. Casiraghi, "Assessment of the sugars and ethanol development in beer fermentation with FT-IR and multivariate curve resolution models," Food Research International, vol. 62, pp. 602-608, 2014.

[41] L. Xie, X. Ye, D. Liu, and Y. Ying, "Quantification of glucose, fructose and sucrose in bayberry juice by NIR and PLS," Food Chemistry, vol. 114, no. 3, pp. 1135-1140, 2009.

[42] H. Shi, Y. Lei, L. Louzada Prates, and P. Yu, "Evaluation of near-infrared (NIR) and fourier transform mid-infrared (ATR-FT/MIR) spectroscopy techniques combined with chemometrics for the determination of crude protein and intestinal protein digestibility of wheat," Food Chemistry, vol. 272, pp. 507-513, 2019.
[43] G. B. Rossi and V. A. Lozano, "Simultaneous determination of quality parameters in yerba mate (Ilex paraguariensis) samples by application of near-infrared (NIR) spectroscopy and partial least squares (PLS)," LWT-food Science and Technology, vol. 8, no. 126, pp. 195229-195239, 2020.

[44] P. Williams and J. Antoniszyn, Near-Infrared Technology: Getting the Best Out of Light, Sun Press, Providence, RI, USA, 2019.

[45] H. E. Smyth, D. Cozzolino, W. U. Cynkar, R. G. Dambergs, M. Sefton, and M. Gishen, "Near infrared spectroscopy as a rapid tool to measure volatile aroma compounds in Riesling wine: possibilities and limits," Analytical and Bioanalytical Chemistry, vol. 390, no. 7, pp. 1911-1916, 2008.

[46] Y. Shao, Y. Cen, Y. He, and F. Liu, "Infrared spectroscopy and chemometrics for the starch and protein prediction in irradiated rice," Food Chemistry, vol. 126, no. 4, pp. 1856-1861, 2011.

[47] S. Shin, Y. Lee, S. Kim, S. Choi, J. G. Kim, and K. Lee, "Rapid and non-destructive spectroscopic method for classifying beef freshness using a deep spectral network fused with myoglobin information," Food Chemistry, vol. 352, Article ID 129329, 2021. 